Florian Part, Sabine Greßler, Marion Huber-Humer, André Gazsó*

\section{Zusammenfassung}

Künstlich hergestellte Nanomaterialien (ENM) können potenziell während aller Abfallbehandlungsprozesse freigesetz† werden sowie in Reststoffen, Altstoffen, Sekundärrohstoffen oder Komposten akkumulieren. Zum Verbleib und Verhalten von ENM während der Abfallverwertung und -entsorgung liegen jedoch erst wenige Untersuchungen vor. In Österreich werden mehr als die Hälfte des in Haushalten anfallenden Abfalls getrennt gesammelt und als Altstoff, biogene Abfälle sowie Problemstoffe und Elektroaltgeräte weiterbehandelt. Der Rest wird entweder in Müllverbrennungsanlagen (MVA) oder in mechanisch-biologischen Abfallbehandlungsanlagen (MBA) behandelt. Erste Untersuchungen in MVAs zeigen, dass sich thermisch stabile ENM (Metalloxide) überwiegend in den festen Rückständen (Schlacke, Flugasche) anreichern. In Österreich werden diese überwiegend in Reststoffdeponien abgelagert. ENM können auch während des Recyclings von Produkten wieder freigesetz† werden (etwa Quantum Dots aus LEDs von Elektroaltgeräten oder CNTs aus Verbundmaterialien). Nanosilber scheint sich beim Recycling negativ auf die mechanischen Eigenschaften von Kunststoffen auszuwirken. ENM können direkt als Produktionsabfälle oder als Bestandteil von "Nano-Produkten" bzw. als Sekundärabfälle, wie ENM-haltige Klärschlamme oder Verbrennungsrückstände, deponiert werden. Es wird geschätzt, dass weltweit zwischen 60 bis $86 \%$ der am häufigsten eingesetzten ENM in Deponien landen. Da die Einsatzgebiete von ENM sehr mannigfaltig sind und deren Schicksal in der Umwelt im Einzelfall sehr unterschiedlich sein kann, können noch keine verallgemeinerten Aussagen getroffen werden.

* Korrespondenzautor

\title{
Umweltrelevante Aspekte von Nanomaterialien am Ende der Nutzungsphase - Teil II: Abfallverwertung und -entsorgung
}

\section{Einleitung}

In vorigen NanoTrust Dossiers wurde bereits allgemein auf mögliche Entsorgungswege von "Nano-Produkten" (siehe dazu') und auf das Umweltverhalten von ENM (Engineered Nanomaterials) während der Abwasserreinigung (siehe dazu' ${ }^{2}$ ) eingegangen. ENM können pozentiell in allen Abfallströmen enthalten sein, da deren Verwendung in Konsumprodukten sehr mannigfaltig ist. Die Einsatzgebiete von ENM reichen bereits von Baustoffen über Kosmetika und Textilien bis hin zu Elektronikgeräten (siehe dazu ${ }^{3}$ ). Hinsichtlich einer möglichen Freisetzung ist es unabdingbar zu unterscheiden, ob ENM in einem Produkt gebunden (z. B. CNT in Kunststoffen) oder ungebunden vorliegen $\left(z . \mathrm{B} \mathrm{TiO}_{2}\right.$ in Sonnenschutzmitteln). Sobald ENM ins urbane Abwassersystem gelangen, stellen Klärschlämme eine wichtige "Barriere" vor deren Freisetzung in die Umwelt dar. Die Entsorgung von diesen Sekundärabfällen ist länderspezifisch sehr unterschiedlich, wobei in Österreich die größten Mengen derzeit in Wirbelschichtöfen thermisch behandelt werden (siehe dazu ${ }^{4}$ ). Abfallwirtschaftliche Prozesse, wie Abwasserbeseitigung und Abfallbehandlung (biologisch, mechanisch, chemisch oder thermisch), können zur Umwandlung von ENM führen. Diese Transformationsprozesse können z. B. zur Bildung von gelösten lonen, Sulfaten oder Oxiden führen. Nach diesen Prozessen stellt sich die Frage, ob diese Formen (chemische Spezies) noch die Größe eines Nanomaterials besitzen (1-100 nm) und nanospezifische Eigenschaften aufweisen. So können sich durch die vorherrschenden Umweltbedingungen in Abwässern Silbernanopartikel (Ag-NP) zu gelösten Silberionen $\left(\mathrm{Ag}^{+}\right)$, schwerlöslichem Silbersulfid $\left(\mathrm{Ag}_{2} \mathrm{~S}\right)$ oder Silberoxid $\left(\mathrm{Ag}_{2} \mathrm{O}\right)$ umwandeln. Die Unterscheidung der Form ist wichtig, um das Umwelt- sowie Aggregationsverhalten von ENM abschätzen zu können. Über das Verhalten von ENM-haltigen Produkten, welche am Ende ihrer Nutzungsdauer sind, ist derzeit noch wenig bekannt. In diesem Dossier wird der aktuelle Stand des Wissens über die Entsorgung von ENM-haltigen (festen) Abfällen überblicksmäßig dargestellt.

Die Behandlungswege von festen Abfällen aus Haushalten und ähnlichen Einrichtungen sind in Abbildung 1 in Form eines Stoffflussdiagramms ersichtlich. Wie sich ENM während der Behandlung von festen Abfällen verhalten können, wird in den folgenden Kapiteln zusammenfassend dargestellt.

\section{Wie verhalten sich ENM bei der Müllverbrennung?}

Vejerano et al. ${ }^{6}$ untersuchten das Verhalten von nano- $\mathrm{TiO}_{2}$, - Nickeloxid (NiO), -Silber $(\mathrm{Ag})$, Ceroxid $\left(\mathrm{CeO}_{2}\right)$ und -Eisenoxid $\left(\mathrm{Fe}_{2} \mathrm{O}_{3}\right)$ sowie von $\mathrm{CdSe} / \mathrm{ZnS}$ Quantum Dots und Fullerenen $\left(\mathrm{C}_{60}\right)$ während der thermischen Verwertung im Labormaßstab. Um die Verbrennung von medizinischen $\mathrm{Ab}$ fällen sowie von Laborrückständen im Labormaßstab simulieren zu können, wurden Abfälle aus Papier, Polyethylenterephthalat (PET) und Polyvinylchlorid (PVC) mit den ausgewählten ENM in unterschiedlichen Massenanteilen vermengt und verbrannt. Ziel dieser Versuche war, den Einfluss der ENM auf den Verbrennungsprozess selbst sowie auf die Feinstaubildung näher zu untersuchen. Die Laborversuche ergaben, dass die Feinstaubbildung durch die eingesetzten ENM-Mengen nicht signifikant beeinflusst wurde, es also zu keiner vermehrten Bildung von Feinstaub kam. Eine 
signifikante Änderung der Anzahlgrößenverteilung bzw. Verschiebung in Bereiche mit kleineren Partikeldurchmessern wurde nur bei unrealistisch hohen ENM-Dosierungen festgestellt. Die Verbrennung von ENM, bestehend aus Metalloxiden, führte sogar zu einer geringeren Bildung von volatilen polycyclischen aromatischen Kohlenwasserstoffen (PAK), welche ein unerwünschtes, weil toxisches, Nebenprodukt aus Verbrennungsprozessen darstellen. Die stärkere katalytische Wirkung dieser ENM $\left(\mathrm{CeO}_{2}, \mathrm{Fe}_{2} \mathrm{O}_{3}\right.$, $\mathrm{NiO}$ und $\mathrm{TiO}_{2}$ ) könnte aufgrund der erhöhten oxidativen Eigenschaften der Partikeloberfläche sowie durch die Oxidationsstufe des Metalls und deren spezifische Eigenschaften hervorgerufen werden. Hingegen können CdSe Quantum Dots zu einer erhöhten Bildung von PAK-Emissionen führen. Die Autoren vermuten, dass dies auf ihre geringe Größe $(<10 \mathrm{~nm})$ und die spezifischen physikochemischen Eigenschaften der Quantum Dots zurückzuführen sei. Durch die hohen Temperaturen $\left(>850^{\circ} \mathrm{C}\right)$, welche in Verbrennungsanlagen erreicht werden, können auch die physikalischen bzw. kristallinen Eigenschaften von Metalloxiden (z. B. nano$\mathrm{Fe}_{2} \mathrm{O}_{3},-\mathrm{TiO}_{2}$ ) und in weiterer Folge ihre toxischen Eigenschaften verändert werden.
Ausgehend von einer mit Rauchgaswäscher, Elektro- und Gewebefilter ausgestatteten Müllverbrennungsanlage nehmen Vejerano et al. ${ }^{7}$ an, dass nur $0,021 \%$ bis $0,25 \%$ der betrachteten ENM in die Atmosphäre entweichen. Die Untersuchung ergab, dass die ENM hauptsächlich in den Verbrennungsrückständen zu finden sind (z. B. Schlacke bzw. Rostasche, Flugasche). In der Schlacke lagen nano- $\mathrm{TiO}_{2},-\mathrm{CeO}_{2},-\mathrm{FeO}_{3}$ in größeren Aggregaten vor, während CdSe Quantum Dots, nano-Ag und -NiO keine größeren Cluster bildeten. Die Autoren wiesen darauf hin, dass Versuche im Großmaßstab durchgeführt werden sollten, um genauere Aussagen treffen zu können.

In der Schweiz wurde bereits ein Großversuch in einer Müllverbrennungsanlage mit einer Jahreskapazität von 200.000 t durchgeführt ${ }^{8}$. Ziel war, den Verbleib und die Massenflüsse von nano- $\mathrm{CeO}_{2}$ unter realen $\mathrm{Be}$ dingungen näher untersuchen zu können. Im Zuge des ersten Versuches wurden $10 \mathrm{~kg}$ nano- $\mathrm{CeO}_{2}$, suspendiert in Wasser, direkt auf ca. 7 † Restmüll aufgebracht. Im zweiten Versuch wurde $1 \mathrm{~kg}$ dieser Tracernanopartikel direkt in den Verbrennungsraum der Rostfeuerungsanlage gesprüht. Anschließend wurden die Konzentrationen von Cerium $(\mathrm{Ce})$ jeweils in der Schlacke, Filterasche, im Prozesswasser sowie in den gereinigten Rauchgasen mittels massenspektrometrischer Messverfahren (ICP-MS ${ }^{9}$ ) bestimmt, um in weiterer Folge eine Massenbilanz für nano- $\mathrm{CeO}_{2}$ erstellen zu können. Das schematische Fließbild der Verbrennungsanlage und die jeweilige Probeentnahmestellen werden in Abbildung 2 dargestellt. Die Großversuche von Walser et al. ${ }^{8}$ ergaben, dass in der Schlacke 81 \% bzw. 53 \% (Versuch 1 bzw. Versuch 2), in der Flugasche $19 \%$ bzw. $45 \%$, im Prozesswasser $0,02 \%$ bzw. 1,7 \% und im gereinigtem Rauchgas nur $0 \%$ bzw. 0,0004 \% der zugegeben Tracer-Mengen aufgefunden wurden. Nanopartikuläres $\mathrm{CeO}_{2}$, welches an der Oberfläche von anderen Verbrennungsrückständen haftete, lag nach weiteren Analysen nicht in gebundener Form vor. Die Autoren gingen davon aus, dass keine chemischen oder physikalischen Veränderungen stattgefunden haben.

In Deutschland untersuchten Liesen et al. ${ }^{10}$ im Rahmen eines Großversuches das Verhalten und die thermische Stabilität von nano- $\mathrm{CeO}_{2}$ in einer Sonderverbrennungsanlage. Hierbei wurde $1 \mathrm{~g} / \mathrm{L} \mathrm{CeO}_{2}$ (suspen-

\section{Behandlung von Abfällen aus Haushalten und ähnlichen Einrichtungen}

In Österreich wurden im Jahre 2009 rund 43 \% der Abfälle, welche aus Haushalten und ähnlichen Einrichtungen stammen, entweder der Müllverbrennung (MVA) oder der mechanisch-biologischen Abfallbehandlung zugeführt (MBA). Nach einer Abtrennung der heizwertreichen Fraktion im Zuge der MBA, wird diese Fraktion wiederum der thermischen Behandlung zugeführt. Die heizwertarme Fraktion aus der MBA wird zuerst biotechnisch behandelt (z. B. verrottet) und anschließend deponiert. Rund $57 \%$ des in Haushalten und ähnlichen Einrichtungen gesammelten Abfalls werden getrennt gesammelt und der Altstoffverwertung (z. B. Kunststoff-Recycling) oder der biotechnischen Verwertung (z. B. Kompostierung oder Biogasproduktion) zugeführt. Problemstoffe und Elektroaltgeräte (EAG) werden ebenso getrennt gesammelt und in Sonderbehandlungsanlagen aufbereitet. Gesamtheitlich betrachtet gehen rund $44,8 \%$ des Gesamtaufkommens bei der thermischen und biotechnischen Verwertung als Masse verloren (z. B. durch Umsetzung zu $\mathrm{CO}_{2}$ oder Wasserdampf). 38,9\% des Gesamtaufkommens aus Haushalten und ähnlichen Einrichtungen werden stofflich verwertet bzw. recycelt, nur 13,8 \% deponiert und $2,5 \%$ werden der Sonderbehandlung zugeführt. Durch Abfallbehandlungsprozesse können ENM potenziell durch mechanische, chemische oder thermische Einwirkungen (unbeabsichtigt) freigesetzt werden oder in bestimmten Abfallströmen, wie Schlacken oder Sekundärrohstoffen aus dem Recycling, akkumulieren.

Abbildung 1:

Verwertung und Beseitigung von Abfällen aus Haushalten und ähnlichen Einrichtungen im Jahr 2009. ENM können potenziell während aller Abfallbehandlungsprozesse

freigesetzt werden sowie in Reststoffen, Altstoffen (Sekundärrohstoffen) oder Komposten akkumulieren.

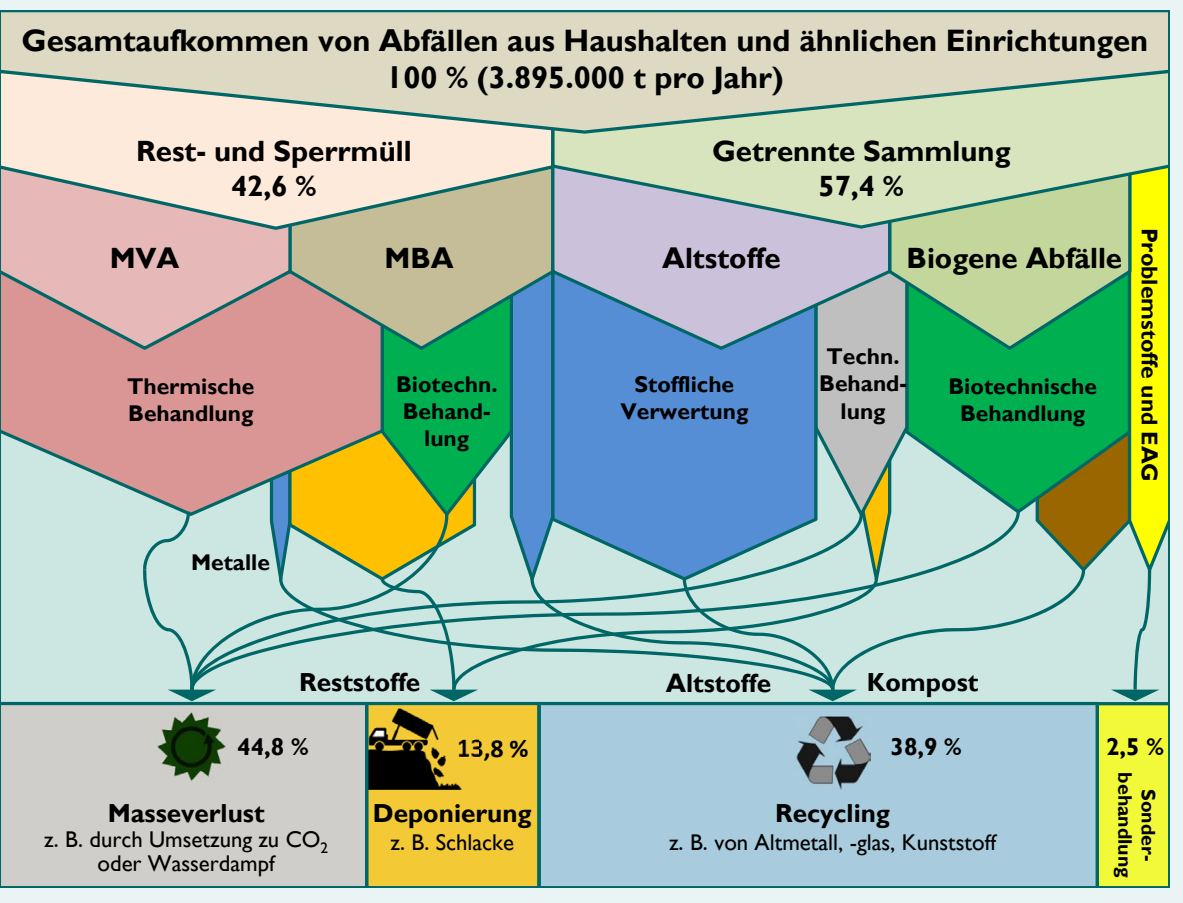


a Case 1: $10 \mathrm{~kg}$ nano- $-\mathrm{CeO}_{2}$ sprayed on waste Case 2: $1 \mathrm{~kg}$ nano- $\mathrm{CeO}_{2}$ sprayed into the furnace

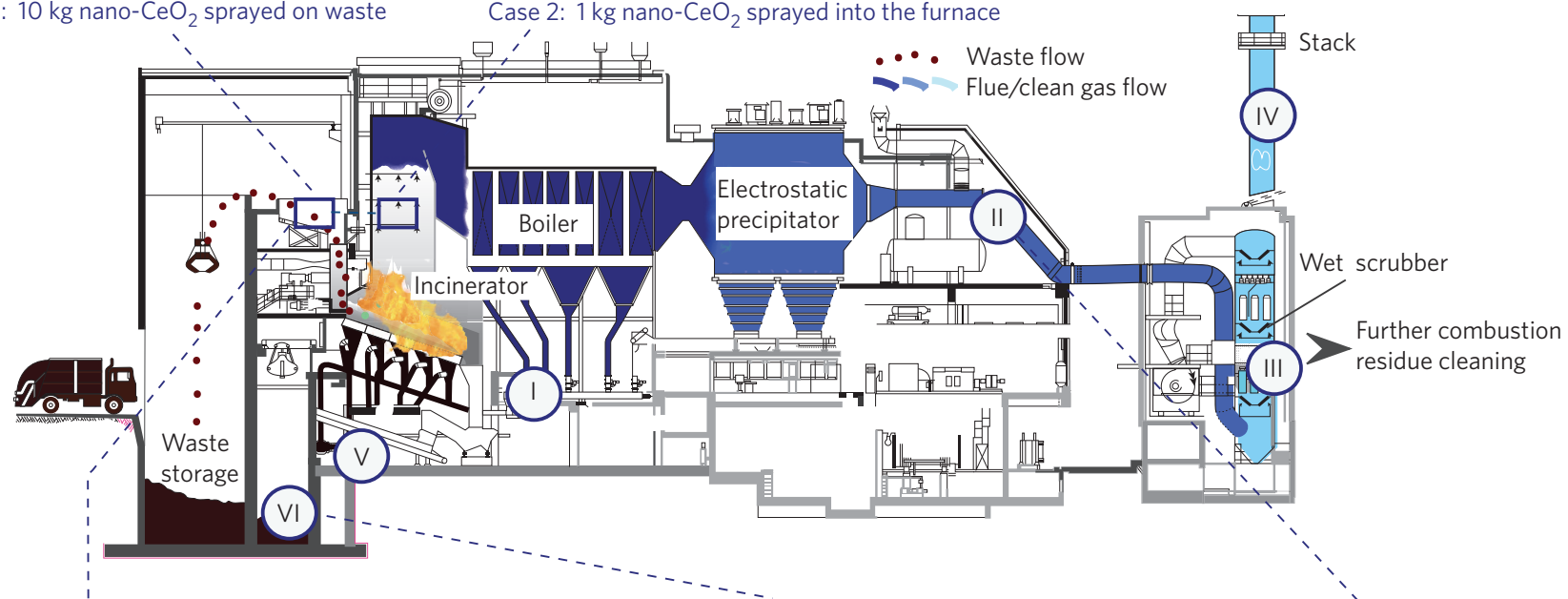

b Nano- $\mathrm{CeO}_{2}$ before incineration

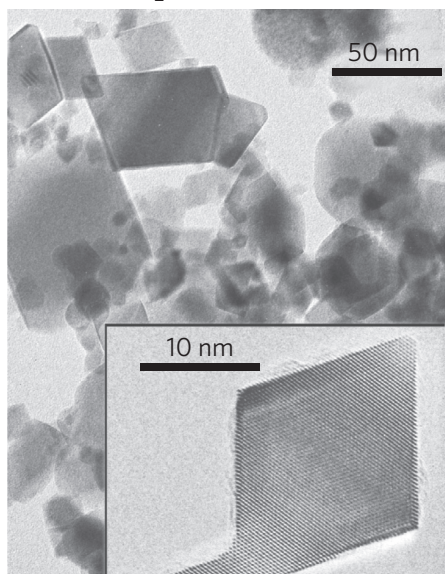

Nano- $\mathrm{CeO}_{2}$ after incineration in the slag
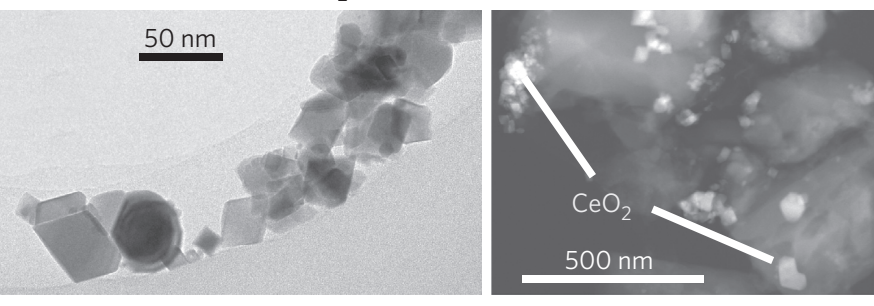

c

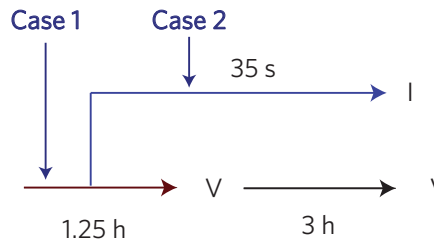

Nano- $\mathrm{CeO}_{2}$ in the flue gas
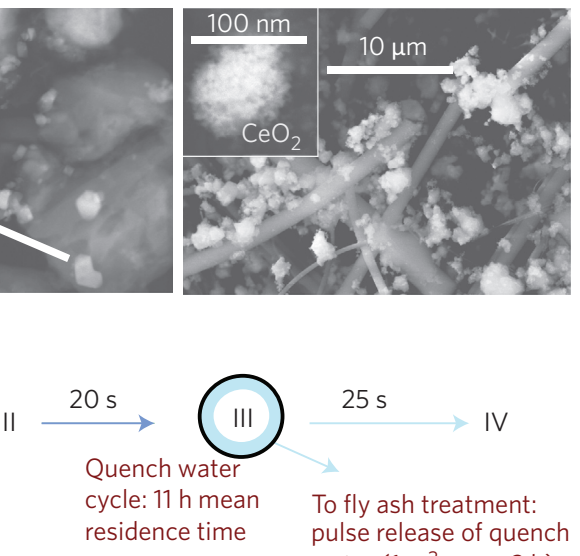

$25 \mathrm{~s}$

IV

To fly ash treatment: pulse release of quench water ( $1 \mathrm{~m}^{3}$ every $\left.2 \mathrm{~h}\right)$

Abbildung 2: Detektion von $\mathrm{CeO}_{2}$-Tracerpartikeln in festen sowie flüssigen Verbrennungsrückständen.

a: $\mathrm{Nano}-\mathrm{CeO}_{2}$ wurde direkt in die Vorkammer des Verbrennungsraumes (Versuch 1) oder in den Rauchgasstrom direkt über dem Verbrennungsraum gesprüht (Versuch 2). Probeentnahmestellen I-VI vor und nach Zugabe von nano- $\mathrm{CeO}_{2}$. b: Aufnahmen mittels Elektronenmikroskopie von kristallinem nano- $\mathrm{CeO}_{2}(10-50 \mathrm{~nm})$ von unterschiedlichen Probeentnahmestellen. c: Schematische Darstellung der erwarteten Verweilzeiten von nano- $\mathrm{CeO}_{2}$ im Rauchgasstrom (Helligkeit der blauen Pfeile soll die Reinheit des Gases darstellen). ${ }^{8}$

diert in Wasser) direkt in die Flamme der Verbrennungskammer injiziert und die Partikelanzahlkonzentration sowie Anzahlgrößenverteilung mittels Aerosolmessgerät (SMPS ${ }^{11}$ ) und Elektronenmikroskopie (TEM ${ }^{12}$ ) bestimmt. Die Untersuchungen zeigten, dass sich nano- $\mathrm{CeO}_{2}$ in der Flamme zu kleineren, einzelnen Primärpartikeln (durch Deagglomeration oder Sublimation), zu größeren Clustern (durch Agglomeration) oder Aggregaten (durch Versinterung) umformen können. Nach der anschließenden Rauchgasreinigung konnten über 99,9\% dieser Tracernanopartikel abgeschieden werden. Demnach ist eine luftseitige diffuse Freisetzung über den Kamin vernachlässigbar gering (Anm.: solange eine adäquate Rauchgasreinigungstechnologie eingesetzt wird).
Somit reichern sich thermisch stabile ENM, wie nano- $-\mathrm{CeO}_{2},-\mathrm{TiO}_{2},-\mathrm{NiO},-\mathrm{CeO}_{2}$ und $-\mathrm{Fe}_{2} \mathrm{O}_{3}$, überwiegend in den festen Rückständen einer Müllverbrennungsanlage (z. B. Schlacke, Flugasche oder feste Rauchgasreinigungsprodukte) an. Diese Rückstände werden in Österreich zum größten Teil in sogenannten Reststoffdeponien abgelagert. Verbrennungsrückstände mit geringer Schadstoffbelastung können aber auch als Ersatzbaustoffe (z. B.: im Straßenbau) stofflich verwertet werden. Vorgaben und Grenzwerte für spezifische Nanopartikel in Abfällen, Reststoffen und Sekundärrohstoffen existieren derzeit in Europa wie auch in Österreich in der Abfallgesetzgebung nicht. Eine Freisetzung von ENM aus Recyclingbaustoffen und anderen Sekundärrohstoffen kann daher nicht ausgeschlossen werden.

\section{Wie verhalten sich ENM während des Recyclings?}

Über das Verhalten von ENM während der stofflichen Verwertung von Abfällen bzw. des Recyclings ist derzeit noch sehr wenig bekannt. Aus Sicht des Arbeitnehmerschutzes wurden bis dato ebenso nur sehr wenige Untersuchungen in Abfallbehandlungsanlagen durchgeführt. ENM, die in einer Produktmatrix in gebundener Form vorliegen, können erst durch mechanische, chemische oder thermische Prozesse wieder freigesetz $\dagger$ werden (siehe dazu ${ }^{13}$ ). So können beispielsweise Quantum Dots in LED-Hintergrundbeleuchtungen während des Recyclings von Elektroaltgeräten wieder freigesetzt werden. Liv et al. ${ }^{14}$ untersuchten das Freisetzungsverhalten von CdSe/ZnS Quantum Dots, die in Beleuchtungssystemen (LEDs oder TVs) 
oder Solarmodulen eingesetzt werden und dort in der einer festen Matrix eingebettet sind. Im Zuge der Laborversuche wurden die Quantum Dots Auslaugungsprozessen mit säurehaltigen Lösungsmitteln ausgesetzt. Hierbei lösten sich nur relativ geringe Konzentrationen hoch toxisch wirkender Cadmiumionen $\left(\mathrm{Cd}^{2+}\right)$. Das Auftreten eines Risikos durch diese Substanzen ist während der Nutzung von Beleuchtungssystemen und Solarmodulen mit Quantum Dots als sehr gering einzuschätzen. Es ist allerdings noch völlig ungeklärt, ob Quantum Dots aus Galliumnitrid (GaN), Indiumphosphid (InP), Cadmiumselenid ( $\mathrm{CdSe}$ ) oder -tellurid ( $\mathrm{CdTe}$ ) durch Aufbereitungsprozesse von derartigen Elektroaltgeräten unbeabsichtigt wieder freigesetzt werden können.

Eine Schweizer Forschergruppe ${ }^{15}$ entwickelte für Kompositmaterialien, die Carbonnanoröhrchen (CNT) enthalten, potenzielle Emissionsszenarien. Die Autoren gehen davon aus, dass eine mögliche CNT-Freisetzung während der Produktion (z. B. durch Vorgänge beim Extrudieren von Kunststoffen) unwahrscheinlich ist. Während der Nutzungsphase können aber z. B. aus CNT-haltige Autoreifen oder Textilien durch Abrieb CNT freigesetzt werden. Eine unbeabsichtigte Freisetzung während des Recyclings von CNT-haltigen Produkten, wie Windräder, Autoreifen, Textilien, Sport- oder Elektronikequipment, kann nach der Einschätzung von Nowack ef al. ${ }^{12}$ durchaus stattfinden.

In einer Studie von Sánchez et al. ${ }^{16}$ wurde der Einfluss von Nanomineralien und nanopartikulärem Calciumcarbonat $\left(\mathrm{CaCO}_{3}\right)$, Silber $(\mathrm{Ag})$, sowie Zinkoxid $(\mathrm{ZnO})$ auf das Recycling von Kunststofffolien untersucht. Hierbei wurden Folien aus Polyethylen (PE), Polypropylen (PP) und Polyethylenterephthalat (PET) mit und ohne ENM auf ihre mechanischen sowie optischen Eigenschaften getestet. Hinsichtlich der Strapazierfähigkeit konnte kein signifikanter Unterschied zwischen den konventionellen und mit ENM versehenen Recyclingkunststoffen festgestellt werden. Ausgenommen davon waren ENMhaltige PET-Verpackungsfolien, wobei sich nano-Ag negativ auf die mechanischen $\mathrm{Ei}$ genschaften der Kunststoffe auswirkte. Eine zu hohe ENM-Dosierung oder Anreicherung in Recyclingkunststoffen kann aber auch dazu führen, dass beispielsweise die Transparenz der Verpackungsfolien herabgesetz† wird. Der Einsatz von Recyclingkunststoffen hängt im Allgemeinen davon ab, ob die hohen hygienischen und technischen Anforderungen an ein Verpackungsmaterial erfüllt werden können bzw. für welche Zwecke diese wieder eingesetzt werden (z. B. für Lebensmittel- oder Baumaterialverpackungen).
Abfallstoffe, welche ursprünglich keine ENM enthielten, können aber auch als Sekundärrohstoff für die gezielte Herstellung von $\mathrm{Na}$ nomaterialien herangezogen werden. So können z. B. aus biogenen Abfällen oder alten Kunststofftragetaschen kohlenstoffhaltige ENM, wie Graphen ${ }^{17}$ oder $\mathrm{CNT}^{18}$, gewonnen werden. In Zukunft könnte eine vermehrte Nutzung von Altkunststoffen als „ENMQuelle" eine wirtschaftlich umsetzbare Recyclingmöglichkeit darstellen. In diesem Zusammenhang wird auch darauf hingewiesen, dass Altkunststoffe, welche nicht der ordentlichen Abfallsammlung zugeführt werden und unabsichtlich in unsere Umwelt gelangen, sich durch mechanische und chemische Prozesse zu nanoskaligen Partikeln umwandeln können (nanopartikuläre Emissionen), die wiederum zu Umweltbeeinträchtigung führen können ${ }^{19}$.

\section{Wie verhalten sich ENM in Deponien?}

Im Allgemeinen können ENM direkt als Produktionsabfälle oder als Bestandteil von "Nano-Produkten" deponiert werden. Ein indirekter Eintrag ist durch die Ablagerung von Sekundärabfällen gegeben (z. B. von Klärschlämmen, Verbrennungsrückständen etc.). Stoffstrommodellierungen ${ }^{20}$ haben gezeigt, dass die größten ENM-Mengen zukünftig in Deponien angereichert werden. Eine wesentliche Rolle spielen hierbei die sehr unterschiedlichen Einsatzgebiete von ENM in diversen Produkten sowie die teilweise sehr länderspezifischen Entsorgungssysteme (siehe dazu ${ }^{21}$ ).

So schätzen Keller et al. ${ }^{17}$, dass weltweit zwischen 60 bis $86 \%$ der am häufigsten eingesetzten ENM ${ }^{22}$ in Deponien landen. Eine Stoffflussmodellierung unter Berücksichtigung des Schweizer Entsorgungssystems ergab, dass weniger als $100 \mathrm{~kg}$ CNT, $5+\mathrm{ZnO}$ und $\mathrm{Ag}$ - sowie $150+\mathrm{TiO}_{2}$-ENM jährlich in Deponien enden ${ }^{23}$. Hierbei wird der größte Massenanteil als Reststoff nach einer thermischen Abfallbehandlung abgelagert, ein geringer Anteil wird als Baurestmassen direkt deponiert. Müller et al. ${ }^{19}$ heben hervor, dass derzeit noch sehr wenig über das Verhalten von ENM in Deponien bekannt ist.

Im Rahmen einer in Frankreich durchgeführten Studie ${ }^{27}$ konnten bereits Nanomaterialien und größere Partikel nachgewiesen werden, die aus Deponien und Abfällen ausgelaugt wurden. Hierbei konnte nicht eindeutig zwischen nanopartikulären Emissio- nen, die von nicht-nanomaterial-haltigen Abfällen stammen und unbeabsichtigt entstehen können, und ENM unterschieden werden. In einer U.S.-amerikanischen Studie ${ }^{28}$ wurde das Verhalten von CNT in Deponien näher untersucht. Die Versuche unter Laborbedingungen zeigten, dass CNT in Deponiesickerwässer kolloidal stabil bleiben können und somit mobil sind, solange gelöste Huminsäuren vorhanden sind. Ebenso stellte die lonenstärke einen entscheidenden Faktor für die potenzielle Mobilität von ENM in Sickerwässern dar. Bolyard et al. ${ }^{29}$ untersuchten den Einfluss und die Auswirkung von ENM (nano- $\mathrm{ZnO},-\mathrm{TiO}_{2}$ und $-\mathrm{Ag}$ ) auf biologische Abbauprozesse, die in realen Sickerwässern stattfinden. Bei diesen Laborversuchen und unter Verwendung von relativ hohen ENM-Konzentrationen $(0,1-100 \mathrm{mg} / \mathrm{L})$ konnten keine signifikanten Veränderungen der ausgewählten Deponieparameter ${ }^{30}$ festgestellt werden. In einer anderen U.S.-amerikanischen Studie ${ }^{31}$ wurden die Auswirkungen von nano-Ag auf mikrobielle Prozesse, welche in Deponien mit einem hohen Anteil an organischen Abfällen stattfinden kön-

\section{Verhalten und Transport} von ENM in Umweltmedien:

Eine mögliche Mobilisierung und Transformation von ENM kann vorwiegend durch hydrodynamische Transportvorgänge (Auswaschung bzw. Deposition), Lösungs-, De- bzw. Adsorptions- sowie durch Oxidations- bzw. Reduktionsprozesse hervorgerufen werden ${ }^{24}$. Verhalten und Verbleib von ENM, z. B. in einer Deponie, sind stark von den Umweltbedingungen, wie $\mathrm{pH}$-Wert, lonenstärke, Gesamtgehalt an organischer Substanz, Gehalt an gelösten Huminsäuren, sowie von der kolloidalen Stabilität der ENM abhängig. Die vorherrschenden Bedingungen variieren wiederum mit Deponietyp und -alter sowie der Betriebsart. Es kann auch nicht ausgeschlossen werden, dass bestimmte ENM künstlich eingerichtete Barrieren wie Deponiebasisabdichtungssysteme durchdringen können ${ }^{25}$. Gelangen ENM ins Grundwasser oder in Oberflächengewässer, können diese - sowie auch natürlich vorkommende Nanomaterialien - zusätzlich als „Trägersubstanzen" für andere Schadstoffe dienen ${ }^{26}$. Das Umweltmonitoring von ENM und assoziierten Schadstoffen stellt generell eine große Herausforderung dar, da eine Unterscheidung zwischen natürlich vorkommenden und künstlich hergestellten Nanomaterialien bei sehr geringen Umweltkonzentrationen sehr aufwendig ist. 
nen, untersucht. Eine Hemmung der Methangasbildung konnte erst bei unrealistisch hohen ENM-Konzentrationen ( $\geq 10 \mathrm{mg}$ / $\mathrm{kg}^{32}$ festgestellt werden.

In den U.S.A. wird der Großteil der Abfälle ohne Vorbehandlung (Abfallverbrennung, Recycling etc.) direkt deponiert. Es werden auch in einigen EU-Staaten weiterhin Abfälle direkt abgelagert (siehe dazu ${ }^{33}$ ). Nähere Untersuchungen zum Verhalten und Verbleib von ENM in Deponien für europäische Verhältnisse gibt es derzeit wenige. In Österreich wird aktuell das Mobilitätsverhalten von kolloidal stabilen ENM unter Berücksichtigung vorherrschender Deponiebedingungen näher untersucht ${ }^{34}$. Unter Verwendung von Oberflächen-modifizierten $\mathrm{CdTe} / \mathrm{ZnS}$ Quantum Dots, die hierbei als Tracernanopartikel ${ }^{35}$ fungieren, versucht man, wichtige Grundlagen hinsichtlich der kolloidalen Stabilität und Mobilität von ENM unter Deponiebedingungen zu schaffen. Im Zuge einer von der EU geförderten Studie ${ }^{36}$ werden Diffusionsexperimente von ENM durchgeführt, um die Durchlässigkeit von Deponiebasisdichtungen beurteilen zu können.

ENM stellen nicht nur eine potenzielle Schadstoffbelastung in der Umwelt dar, sondern können auch zur Altlastensanierung oder Trinkwasseraufbereitung verwendet werden. So gibt es derzeit ein laufendes EU-Forschungsprojekt ${ }^{37}$, wobei der Einsatz von nullwertigem Nanoeisen zur Schadstoffentfrachtung bei kontaminierten Standorten untersucht wird. Ziel dieses Projektes ist, eine kostengünstige Sanierungsmethode für sogenannte Altlasten, wie alte Industriestandorte oder Deponien, zu entwickeln. Mittels Nanoeisen können Grundwässer, welche mit organischen oder anorganischen Schadstoffen (z. B. halogenierte Methane, polychlorierte Kohlenwasserstoffe oder Schwermetalle) belastet sind, saniert werden ${ }^{38}$.

\section{Fazit}

In diversen internationalen Studien durchgeführte Stoffflussmodellierungen zeigen, dass die End-of-Life-Phase von "Nano-Produkten" hinsichtlich der potenziellen Freisetzung von ENM eine wesentliche Rolle einnehmen kann. Über das Verhalten und den Verbleib von ENM in Abfallströmen, insbesondere in Deponien, ist derzeit aber noch wenig bekannt. In Zukunft müssen noch weitere Untersuchungen durchgeführt werden, um allgemeinere Aussagen über das Verhalten von ENM in Abfallströmen und Deponien ableiten zu können. Derzeit bleiben noch viele Fragen hinsichtlich des Verbleibs ("Senkenwirkung") und einer möglichen Freisetzung von nanopartikulären Emissionen in den unterschiedlichen Abfallbehandlungsprozessen (z. B. während des Recyclings) sowie hinsichtlich Verhalten und Transport von ENM in Reststoffen auf Deponien offen. Es müssen vor allem Messmethoden (weiter-)entwickelt werden, um ENM-haltige Abfälle und nanopartikuläre Emissionen, wie Ultrafeinstäube in Abfallbehandlungsanlagen oder gelöste Nanopartikel im Deponiesickerwasser, detektieren und charakterisieren zu können. So können, z. B. beim Recycling von Elektroaltgeräten, Baustoffen oder Kunststoffen, sowohl ENM freigesetzt werden, aber auch unbeabsichtigt nanopartikuläre Emissionen aus konventionellen Produkten (ohne ENM) entstehen. Allerdings liegen für Recyclingprozesse derzeit keine näheren Untersuchungen mit geeigneten Messmethoden vor. Ein verlässliches Umweltmonitoring ist in Zukunft unabdingbar, um (langfristige) Umweltauswirkungen von ENM während und nach deren Nutzungsphase beurteilen zu können.

\section{Anmerkungen und Literaturhinweise}

1 NanoTrust Dossier Nr. 040.

2 NanoTrust Dossier Nr. 043.

3 NanoTrust Dossier Nr. 041.

4 NanoTrust Dossier Nr. 043.

5 Eigene Darstellung basierend auf Daten aus dem Bundes-Abfallwirtschaftsplan (BAWP) 2011 (Teil I), Seite 42, BMLFUW.

6 Vejerano, Eric P., et al., 2013, Emissions of Polycyclic Aromatic Hydrocarbons, Polychlorinated Dibenzo-p-Dioxins, and Dibenzofurans from Incineration of Nanomaterials, Environmental Science \& Technology 47(9), 48664874. http://dx.doi.org/10.1021/es304895z.

7 Vejerano, Eric P., et al., 2014, Characterization of particle emissions and fate of nanomaterials during incineration, Environmental Science: Nano 1(2), 133-143. http://dx.doi.org/10.1039/C3EN00080J.

8 Walser, Tobias, et al., 2012, Persistence of engineered nanoparticles in a municipal solidwaste incineration plant, Nat Nano 7(8), 520 524.

http://dx.doi.org/10.1038/nnano.2012.64.

9 ICP-MS ... Massenspektrometrie mit induktiv gekoppeltem Plasma (z. B.: zur Bestimmung der Massenkonzentration).

10 Liesen, I.-M., et al., 2014, Freisetzung von Nanopartikeln bei der thermischen Abfallentsorgung - Stabilität von Nanopartikeln in Flammen, in: Anke Brockeis/Martin Faultich/Sabine Flamme/Martin Kranert/Michael Nelles/ Gerhard Rettenberger/Vera Susanne Rotter, 4. Wissenschaftskongress Abfall- und Ressourcenwirtschaft. 27. und 28. März 2013 in Münster: Deutsche Gesellschaft für Abfallwirtschaft e.V.
11 SMPS ... Scanning Mobility Particle Sizer (SMPS) zur Bestimmung der Partikelanzahlkonzentration und Anzahlgrößenverteilung von Aerosolen wie Ultrafeinstäube.

12 Transmissionselektronenmikroskopie zur Bestimmung der Partikelgröße, -größenverteilung und -form.

13 NanoTrust Dossier Nr. 040.

14 Liu, Jingyu, et al., 2012, Degradation Products from Consumer Nanocomposites: A Case Study on Quantum Dot Lighting, Environmental Science \& Technology 46(6), 3220-3227. http://dx.doi.org/10.1021/es204430f.

15 Nowack, Bernd, et al., 2013, Potential release scenarios for carbon nanotubes used in composites, Environment International 59(0), 1-11. http://www.sciencedirect.com/science/article/ pii/S0160412013000834.

16 Sánchez, C., et al., 2014, Recyclability assessment of nano-reinforced plastic packaging Waste Management 34(12), 2647-2655. http://www.sciencedirect.com/science/article/ pii/S0956053X14003614.

17 Suryawanshi, Anil, et al., 2014, Large scale synthesis of graphene quantum dots (GQDs) from waste biomass and their use as an efficient and selective photoluminescence on-offon probe for $\mathrm{Ag}+$ ions, Nanoscale 6(20), 11664-11670.

http://dx.doi.org/10.1039/C4NR02494J.

18 Altalhi, Tariq, et al., 2013, Synthesis of well-organised carbon nanotube membranes from non-degradable plastic bags with tuneable molecular transport: Towards nanotechnological recycling, Carbon 63(0), 423-433. http://www.sciencedirect.com/science/article/ pii/S0008622313006246. 
19 Besseling, Ellen, et al., 2014, Nanoplastic Affects Growth of S. obliquus and Reproduction of D. magna, Environmental Science \& Technology.

http://dx.doi.org/10.1021/es503001d.

20 Caballero-Guzman, Alejandro, et al., 2015, Flows of engineered nanomaterials through the recycling process in Switzerland, Waste Management 36(0), 33-43.

http://www.sciencedirect.com/science/article/ pii/S0956053X14005236.

Keller, Arturo A./Lazareva, Anastasiya, 2014 Predicted Releases of Engineered Nanomaterials: From Global to Regional to Local, Environmental Science \& Technology Letters 1(1), 65-70. http://dx.doi.org/10.1021/ez400106t. Sun, Tian Yin, et al., 2014, Comprehensive probabilistic modelling of environmental emissions of engineered nanomaterials, Environmental Pollution 185(0), 69-76.

http://www.sciencedirect.com/science/article/ pii/S0269749113005241.

21 NanoTrust Dossier Nr. 040.

22 Laut einer Marktstudie aus dem Jahr 2013 werden folgende ENM am häufigsten in Produkten eingesetzt: $\mathrm{SiO}_{2}, \mathrm{CeO}_{2}, \mathrm{CNT}$, Nanominerale, $\mathrm{Al}_{2} \mathrm{O}_{3}, \mathrm{Cu}, \mathrm{Fe}, \mathrm{ZnO}, \mathrm{TiO}_{2}$ und $\mathrm{Ag}$ (Quelle: The Global Market for Nanomaterials 2002-2016: Production Volumes, Revenues and End Use Markets, 2013, Future Markets, Inc.: 2012; S. 371.

http://www.futuremarketsinc.com/.

23 Mueller, Nicole C., et al., 2013, Modeling the flows of engineered nanomaterials during waste handling, Environmental Science: Processes \& Impacts 15(1), 251-259.

http://dx.doi.org/10.1039/C2EM30761H.

24 Batley, G. E./Mclaughlin, M. J., 2010, Fate of Manufactured Nanomaterials in the Australian Environment: Department of the Environment, Water, Heritage and the Arts.

http://www.clw.csiro.au/publications/science/ 2010/FMF-manufactured-nanomaterials.pdf.
25 Reinhart, Debra R., et al., 2010, Emerging contaminants: Nanomaterial fate in landfills, Waste Management 30(11), 2020-2021.

http://www.sciencedirect.com/science/article/ pii/S0956053X10003855.

26 Hofmann, Thilo, von der Kammer, Frank, 2009, Estimating the relevance of engineered carbonaceous nanoparticle facilitated transport of hydrophobic organic contaminants in porous media, Environmental Pollution 157(4), 1117-1126. http://www.sciencedirect.com/ science/article/pii/S0269749108005782.

Plathe, Kelly L., et al., 2013, The role of nanominerals and mineral nanoparticles in the transport of toxic trace metals: Field-flow fractionation and analytical TEM analyses after nanoparticle isolation and density separation, Geochimica et Cosmochimica Acta 102(0), 213-225.

http://www.sciencedirect.com/science/article/ pii/S0016703712006114.

27 Hennebert, Pierre, et al., 2013, Experimental evidence of colloids and nanoparticles presence from 25 waste leachates, Waste Management 33(9), 1870-1881.

http://www.sciencedirect.com/science/article/ pii/S0956053X13002067.

28 Lozano, Paula/Berge, Nicole D., 2012, Single-walled carbon nanotube behavior in representative mature leachate, Waste Management 32(9), 1699-1711.

http://www.sciencedirect.com/science/article/ pii/S0956053X12001304.

29 Bolyard, Stephanie C., et al., 2013, Behavior of Engineered Nanoparticles in Landfill Leachate, Environmental Science \& Technology 47(15), 8114-8122.

http://dx.doi.org/10.1021/es305175e.

30 In den U.S.A. können der sogenannte biologische Saverstoffbedarf nach fünf Tagen (BSB5) sowie das biochemische Methangaspotenzial (BMP) zur Beurteilung der biologischen Abbaubarkeit von Sickerwässer herangezogen werden.
31 Yang, Yu, et al., 2012, Nanosilver impact on methanogenesis and biogas production from municipal solid waste, Waste Management 32(5), 816-825. http://www.sciencedirect. com/science/article/pii/ S0956053X12000232.

32 Zum Vergleich ist laut den Abschätzungen von Mueller et al. für die Schweiz eine Konzentration von maximal ca. $1 \mathrm{mg}$ nano-Ag pro kg Schlacke zu erwarten.

33 NanoTrust Dossier Nr. 040.

34 Flatscher, A. [Hrsg.], 2013, Bauzwerge, S. 32, Bau- und Immobilien Report, Ausgabe 11/ 2013, Report Verlag GmbH \& Co KG, http://www.report.at/component/ docindexer $/$ ?task $=$ download\&id $=37$

35 Part, F., Zaba, C., Sinner, E.-K., Huber-Humer, M.,2014, Traceability of Quantum Dots in Mature Landfill Leachate in Abstract Proceeding of the $8^{\text {th }}$ Intercontinential Landfill Research Symposium 2014, at Crystal River, Florida.

36 Zuin, S., Massari, A., Motellier, S., Golanski, S., Sicard, Y., 2013, "Nanowaste" management: NanoHouse Dissemination report $N^{\circ} 2013$ 05. http://www-nanohouse.cea.fr/home/ liblocal/docs/Dissemination\%20Reports/ NanoHouse\%20DR5.pdf.

37 NanoRem project, grant agreement No. 309517, Nanotechnology for Contaminated Land Remediation. http://www.nanorem.eu.

38 Apel, P., Becker, H., Dubbert, W., Kabardin, B., Rechenberg, B., Schwirn, K., Völker, D., Winde, C., 2012, Einsatz von Nanoeisen bei der Sanierung von Grundwasserschäden, Langfassung, Datenblatt Nanoprodukte, Umweltbundesamt, Dessau-Roßlau,

http://www.umweltbundesamt.de/sites/ default/files/medien/378/publikationen/ datenblatt_einsatz_von_nanoeisen_bei_der sanierung von grundwasserschaedenlangfassung_dubbert.pdf.

\section{IMPRESSUM:}

Medieninhaber: Österreichische Akademie der Wissenschaften; Juristische Person öffentlichen Rechts (BGBI 569/1921 idF BGBI I 130/2003); Dr. Ignaz Seipel-Platz 2, A-1010 Wien

Herausgeber: Institut für Technikfolgen-Abschätzung (ITA); Strohgasse 45/5, A-1030 Wien;

www.oeaw.ac.at/ita

Erscheinungsweise: Die NanoTrust-Dossiers erscheinen unregelmäßig und dienen der Veröffentlichung der Forschungsergebnisse des Instituts für Technikfolgen-Abschätzung im Rahmen des Projekts NanoTrust. Die Berichte werden ausschließlich über das Internetportal „epub.oeaw” der Öffentlichkeit zur Verfügung gestellt: epub.oeaw.ac.at/ita/nanotrust-dossiers/

NanoTrust-Dossier Nr. 044, April 2015: epub.oeaw.ac.at/ita/nanotrust-dossiers/dossier044.pdf

ISSN: 1998-7293 